\title{
xGASS: Cold gas content and quenching in galaxies below the star forming main sequence
}

\author{
Steven Janowiecki, ${ }^{1,2 \star}$ Barbara Catinella, ${ }^{1,3}$ Luca Cortese, ${ }^{1,3}$ Amelie Saintonge, ${ }^{4}$ \\ Jing Wang 5 \\ ${ }^{1}$ International Center for Radio Astronomy Research (ICRAR), M468, The University of Western Australia, 35 Stirling Highway, Crawley, WA, 6009, Australia \\ ${ }^{2}$ University of Texas, Hobby-Eberly Telescope, McDonald Observatory, TX 79734, USA \\ ${ }^{3}$ ARC Centre of Excellence for All Sky Astrophysics in 3 Dimensions (ASTRO 3D) \\ ${ }^{4}$ Department of Physics and Astronomy, University College London, Gower Street, London WC1E 6BT, UK \\ ${ }^{5}$ Kavli Institute for Astronomy and Astrophysics, Peking University, Beijing 100871, China
}

Accepted 2020 January 13. Received 2020 January 3; in original form 2019 April 12

\begin{abstract}
We use $\mathrm{HI}$ and $\mathrm{H}_{2}$ global gas measurements of galaxies from xGASS and xCOLD GASS to investigate quenching paths of galaxies below the star formation main sequence (SFMS). We show that the population of galaxies below the SFMS is not a 1:1 match with the population of galaxies below the $\mathrm{HI}$ and $\mathrm{H}_{2}$ gas fraction scaling relations. Some galaxies in the transition zone (TZ) 1-sigma below the SFMS can be as HI-rich as those in the SFMS, and have on average longer gas depletion timescales. We find evidence for environmental quenching of satellites, but central galaxies in the TZ defy simple quenching pathways. Some of these so-called "quenched" galaxies may still have significant gas reservoirs and be unlikely to deplete them any time soon. As such, a correct model of galaxy quenching cannot be inferred with SFR (or other optical observables) alone, but must include observations of the cold gas. We also find that internal structure (particularly, the spatial distribution of old and young stellar populations) plays a significant role in regulating the star formation of gas-rich isolated TZ galaxies, suggesting the importance of bulges in their evolution.
\end{abstract}

Key words: galaxies: evolution - galaxies: star formation - galaxies:ISM

\section{INTRODUCTION}

Observations have shown a strong correlation between global estimates of star formation rate (SFR) and stellar mass $\left(\mathrm{M}_{*}\right)$ for populations of galaxies, commonly referred to as the "star forming main sequence" (SFMS, Brinchmann et al. 2004; Elbaz et al. 2007; Salim et al. 2007). The tightness of the SFMS suggests that star formation proceeds in a fairly universal and relatively calm mode, without significant deviations in SFR (Noeske et al. 2007). In an idealized completely isolated galaxy, secular evolution will produce this smooth and gradual evolution as stellar mass grows, gas is consumed, star formation diminishes, and optical color becomes redder. Here, the SFR would depend largely on the available gas, and the two would decline hand-in-hand until the galaxy becomes red and fully dead.

In reality, galaxy evolution is more complex, as most galaxies do not spend their entire lives in isolation. They

^ E-mail: steven.janowiecki@icrar.org (SJ) can merge or interact with other galaxies or dark matter halos, which open up further evolutionary pathways and can have dramatic effects on their gas content and star formation. However, the tightness of the SFMS across cosmic time (e.g., Daddi et al. 2007; Magdis et al. 2010; Whitaker et al. 2012) puts a strong constraint on the maximum amplitude of deviations a star-forming galaxy can experience. In particular, the shape and redshift evolution of the SFMS are consistent with a picture where star formation is regulated by cold gas availability, star formation efficiency ( $\left.\mathrm{SFE}=\mathrm{SFR} / \mathrm{M}_{\text {gas }}\right)$, and feedback (Bouché et al. 2010; Lilly et al. 2013; Saintonge et al. 2016).

As galaxies descend from the SFMS towards the red sequence (RS) they cross through a transitional zone (TZ), historically referred to as the "green valley" because of their intermediate optical and ultra-violet (UV) colors (Martin et al. 2007; Wyder et al. 2007; Salim et al. 2007). However, this transitional population of galaxies is better described by its intermediate specific star formation rates $\left(\mathrm{sSFR}=\mathrm{SFR} / \mathrm{M}_{*}\right)$, rather than the original mass- and dust-dependent color selection criteria (Cortese 2012; Woo et al. 2013; Salim 2014). 
Most of the quenching studies discussed above have relied on UV, optical, near-infrared (NIR), and spectroscopic observations of stellar populations to characterize the evolutionary pathways between the SFMS and the red sequence. However, observations of the neutral atomic (HI) and molecular $\left(\mathrm{H}_{2}\right)$ cold gas reservoirs are required to fully understand the evolutionary potential of galaxies below the SFMS. Even if its stellar populations appear "quenched," a galaxy may possess a significant gas reservoir and still have significant potential for further evolution or growth.

Previous studies of cold gas in the TZ are relatively few in number, as it becomes difficult to detect in galaxies below the SFMS. Cortese \& Hughes (2009) found that TZ galaxies are HI-deficient in the high-density environment of the Virgo Cluster and HI-rich in lower density environments. Independent of environment, Schiminovich et al. (2010) found that the average $\mathrm{HI}$ gas depletion time $\left(\mathrm{M}_{\mathrm{HI}} / \mathrm{SFR}\right)$ was nearly constant on and below the SFMS (although with a large scatter), suggesting a common regulator of both gas supply and star formation. More recently, Saintonge et al. (2016) found a fairly constant SFE (inverse of a depletion time) for massive galaxies in the SFMS, and argued that their overall cold gas supply drives their location in the SFR-M $\mathrm{M}_{*}$ plot, and that their evolution is not driven by bottlenecks in the conversion from atomic to molecular gas.

In this work we quantify the cold gas properties of galaxies in the TZ below the SFMS to characterize their evolutionary pathways. This effort is made possible with extensive $\mathrm{HI}$ observations from the recently completed low-mass extensions of the GALEX Arecibo SDSS Survey (xGASS, Catinella et al. 2018) and its molecular gas counterpart, CO Legacy Database for GASS (xCOLD GASS, Saintonge et al. 2017). Combined, these surveys include measurements of the cold atomic and molecular gas content in a representative, stellar mass-selected sample of galaxies, including a significant number from the gas-poor regime. By including the cold gas reservoirs in galaxies across the SFR-M $\mathrm{M}_{*}$ plane, we consider the potential for future star formation in galaxies that might currently appear "quenched" in terms of their star formation. While these TZ galaxies appear to be in the gloaming of their lives, many have significant reservoirs of cold gas that can support sustained or increased future star formation, and have not been truly quenched yet.

This paper is organized as follows: In Section 2 we quantify the SFMS and gas fraction scaling relations of the xGASS sample. Section 3 quantifies the gas properties in galaxies departing from the SFMS with extreme depletion times. Section 4 characterizes the population of galaxies in the TZ below the SFMS, and Section 5 discusses these results. Finally, we summarize our main results and discuss future work in Section 6.

\section{2 xGASS}

We use galaxies from the xGASS sample (Catinella et al. 2018), which is the extension to lower stellar masses of the sample from Catinella et al. (2010, 2013). xGASS includes $\sim 1200$ galaxies in the local Universe $(0.01<z<0.05)$, evenly sampling the stellar mass interval $10^{9}<\mathrm{M}_{*} / \mathrm{M}_{\odot}<10^{11.5}$ with no other selection criteria. The galaxies have been observed in $21 \mathrm{~cm}$ by Arecibo until $\mathrm{HI}$ is detected or until an up- per limit of a few percent is reached on the HI gas fraction $\left(\mathrm{M}_{\mathrm{HI}} / \mathrm{M}_{*}\right)$. Practically, the gas fraction limit varies as a function of stellar mass (see Section 2.1.1 of Catinella et al. 2018), and galaxies with Hi detections below this gas fraction limit are considered non-detections in this work. This survey represents the most sensitive HI observations of a local representative galaxy sample to date.

In addition to the neutral atomic $21 \mathrm{~cm}$ observations, $\mathrm{CO}(1-0)$ observations exist for $\sim 40 \%$ of xGASS galaxies and are included in xCOLD GASS (Saintonge et al. 2017), which is the extension to lower stellar masses of the sample from Saintonge et al. (2011). Combining these molecular observations with the $\mathrm{HI}$ data provides a complete inventory of the cold gas fuel for star formation throughout the xGASS sample.

Beyond these observations of cold gas, the xGASS sample has a rich set of complementary data. SDSS optical photometry come from Data Release 7 (DR7, Abazajian et al. 2009), and stellar masses come from the value-added catalog ${ }^{1}$ provided by the Max Planck Institute for Astrophysics (MPA) and Johns Hopkins University (JHU), which assume a Chabrier (2003) initial mass function. Further multi-wavelength observations include images in ultra-violet (UV) from the Galaxy Evolution Explorer (GALEX, Martin et al. 2005; Morrissey et al. 2007) and in mid-infrared (MIR) from the Wide-field Infrared Survey Explorer (WISE, Wright et al. 2010). As described in Janowiecki et al. (2017), these UV+MIR observations are used to derive total UV+IR SFRs for xGASS galaxies, fully accounting for the direct emission of the young stellar populations in UV, and for the re-processed dust emission in MIR.

In this work we use these UV+IR SFRs to define the xGASS SFMS and determine the position of each galaxy above or below this relation. Analogously, we use the HI and $\mathrm{H}_{2}$ scaling relations from Catinella et al. (2018) and Saintonge et al. (2017) to quantify how far above or below the typical gas scaling relations galaxies are at a given stellar mass. The details of our SFMS determination and the gas fraction scaling relations we use are described in the following subsections.

\subsection{Fitting the SFMS}

In order to explore galaxies that depart from the SFMS, we must first define it in our sample. The xGASS SFMS was first presented in Catinella et al. (2018) (Equation 2); we discuss its derivation in more detail in this work. The left panel of Figure 1 shows sSFR as a function of stellar mass for the xGASS sample. We include 100 galaxies that have unreliable Hi measurements due to possible source confusion, although they will be excluded from any subsequent analysis that requires Hi masses (see Appendix A in Catinella et al. 2018, for further discussion of source confusion). We are interested in characterizing the SFMS relation as well as its width, to determine how far a galaxy has departed from it. In the following paragraphs, we describe our ex-

\footnotetext{
${ }^{1}$ http://www.mpa-garching.mpg.de/SDSS/DR7/; we used the improved stellar masses from http://home.strw.leidenuniv.nl/ jarle/SDSS/
} 


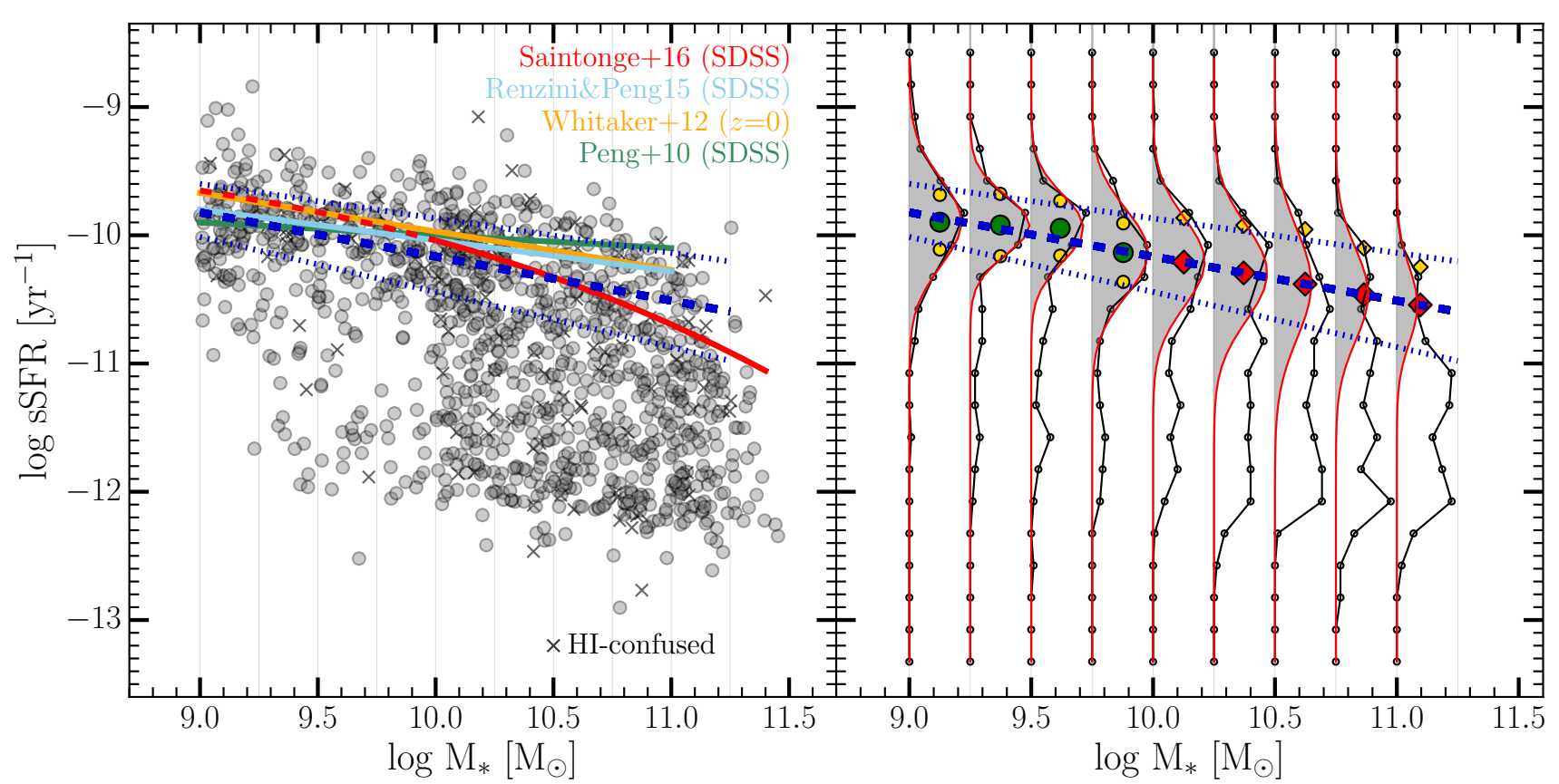

Figure 1. Left panel: sSFR is plotted against stellar mass for our sample (grey circles), including HI-confused galaxies (grey crosses). Blue thick dashed and dotted lines show our SFMS relation and its $1 \sigma$ scatter, and solid colored lines show SFMS relations from other groups. Right panel: a visual summary of our SFMS fitting method. Within each stellar mass bin, black lines and points show the sSFR distributions. Grey histograms show the best-fitting Gaussians in each bin. The four green dots show the modes of the unconstrained Gaussian fits at low masses, and the red diamonds show the extrapolation to higher stellar mass. The yellow symbols show the best-fitting 1-sigma widths in each bin. Our best-fitting SFMS relation $( \pm 1 \sigma)$ is shown as a blue dashed (dotted) line(s).

trapolated half-mirrored Gaussian method to determine the xGASS SFMS and its width.

First, we divide the sample into 0.25 dex-wide stellar mass bins starting from $\mathrm{M}_{*}=10^{9} \mathrm{M}_{\odot}$, as separated by the thin grey vertical lines in the left panel of Figure 1. In each of the four lowest bins $\left(9 \leq \log \mathrm{M}_{*} / \mathrm{M}_{\odot} \leq 10\right)$, the sample is dominated by star-forming galaxies, so we fit unconstrained Gaussians to the sSFR distributions. Using a linear extrapolation of the modes from these four low mass bins, we fit Gaussians to the higher mass bins with a fixed center and allow only their widths to vary. In this higher mass bin we fit a 1-sided Gaussian distribution to only the galaxies that lie above the SFMS to avoid being affected by the increasingly dominant red sequence. After this Gaussian fitting is complete, we fit the $\pm 1 \sigma$ values with a simple linear relation to parametrize the width of the SFMS as a function of stellar mass. Our best-fitting SFMS and its width $(\sigma$, in dex) are given in the form:

$\log \frac{\mathrm{SFR}_{\mathrm{MS}}}{\mathrm{M}_{*}}\left[\mathrm{yr}^{-1}\right]=m_{\mathrm{SFMS}}\left(\log \frac{\mathrm{M}_{*}}{\mathrm{M}_{\odot}}-9\right)+b_{\mathrm{SFMS}}$

$\sigma_{\mathrm{MS}}[\mathrm{dex}]=m_{\mathrm{SFS}}\left(\log \frac{\mathrm{M}_{*}}{\mathrm{M}_{\odot}}-9\right)+b_{\mathrm{SFS}}$.

where $m_{\mathrm{SFMS}}=-0.344 \pm 0.101, b_{\mathrm{SFMS}}=-9.822 \pm 0.057$, $m_{\mathrm{SFS}}=0.088 \pm 0.028$, and $b_{\mathrm{SFS}}=0.188 \pm 0.036$.

Other determinations of the SFMS are also shown in the left panel of Figure 1 and are broadly consistent with ours, although some divergence is seen at higher masses where the SFMS becomes less populated. Each SFMS comes from a different sample of galaxies and uses a different SFR indicator (or indicators), different definitions of star-forming galax- ies, and different fitting methods. While all formulations are similar, we adopt the xGASS SFMS for consistency with our previous work.

We define distance above/below the SFMS as $\triangle$ SFMS:

$\Delta \mathrm{SFMS}=\log \frac{\mathrm{SFR}}{\mathrm{M}_{*}}\left[\mathrm{yr}^{-1}\right]-\log \frac{\mathrm{SFR}_{\mathrm{MS}}}{\mathrm{M}_{*}}\left[\mathrm{yr}^{-1}\right]$.

Note that this difference is essentially a (logarithmic) ratio of two sSFRs and as such is dimensionless. We determine $\triangle$ SFMS in dimensionless logarithmic (dex) units without regard for the changing width of the SFMS as a function of $\mathrm{M}_{*}$. While the SFMS is narrowest ( $\left.0.19 \mathrm{dex}\right)$ at our lowest stellar masses and gets broader with increasing mass, the change is relatively small. Further, using dex units makes it easier to interpret $\triangle \mathrm{SFMS}$ values at different stellar masses in a physically meaningful way.

\subsection{Cold gas fraction relations of SFMS galaxies}

Next we apply a similar approach to characterize the typical $\mathrm{HI}$ and $\mathrm{H}_{2}$ gas fraction scaling relations for the galaxies in the SFMS we have just defined. We carry out a linear fit to the median gas fraction in each bin of stellar mass for galaxies in the SFMS. These binned averages (orange dots) and best-fit relations (black lines) are shown in the bottom two panels of Figure 2, and are parameterized in the same form as the SFMS, as:

$\log \frac{\mathrm{M}_{\mathrm{HI}, \mathrm{MS}}}{\mathrm{M}_{*}}=m_{\mathrm{GFMS}}\left(\log \frac{\mathrm{M}_{*}}{\mathrm{M}_{\odot}}-9\right)-b_{\mathrm{GFMS}}$

$\log \frac{\mathrm{M}_{\mathrm{H}_{2}, \mathrm{MS}}}{\mathrm{M}_{*}}=m_{\mathrm{GF} 2 \mathrm{MS}}\left(\log \frac{\mathrm{M}_{*}}{\mathrm{M}_{\odot}}-9\right)-b_{\mathrm{GF} 2 \mathrm{MS}}$. 

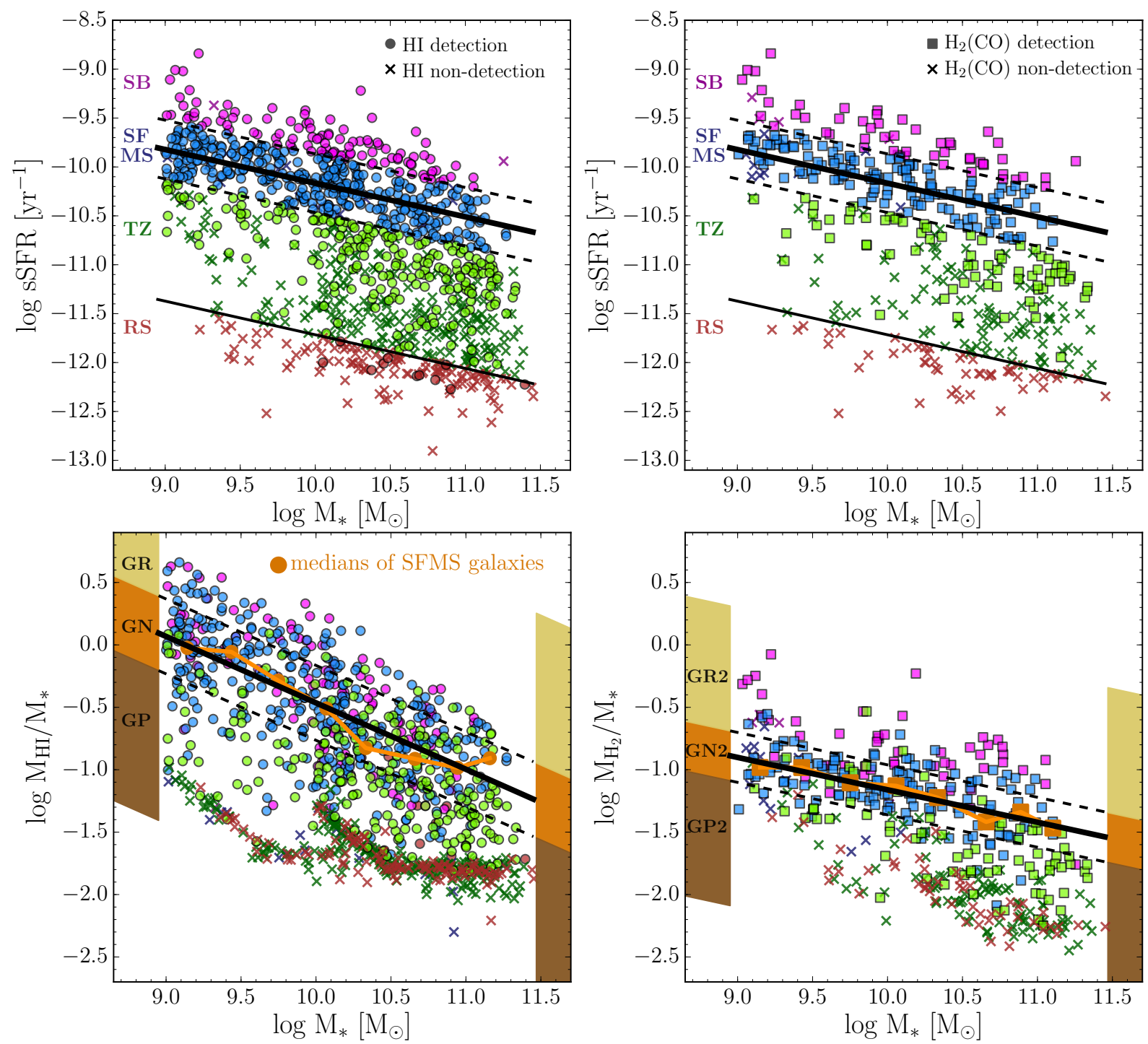

Figure 2. Top two panels: sSFR vs. $M_{*}$ is color coded by position above/on/below the SFMS, with shapes based on their Hi (left panel, circles) or $\mathrm{CO}$ (right panel, squares) detection status, where crosses indicate non-detections. Galaxies with potentially confused HI observations are not included. Black lines show the xGASS SFMS with \pm 0.3 dex dashed lines and the threshold of the RS. Bottom two panels: $\mathrm{HI}$ and $\mathrm{H}_{2}$ gas fractions use the same colors and shapes as the top panels. The large orange points show median $\mathrm{HI}_{\mathrm{I}}$ and $\mathrm{H}_{2}$ gas fractions of galaxies from the SFMS in bins of stellar mass, black lines show linear fits to those averages, and dashed lines show \pm 0.3 dex and \pm 0.2 dex for $\mathrm{HI}$ and $\mathrm{H}_{2}$ gas fractions, respectively.

where $m_{\mathrm{GFMS}}=-0.53 \pm 0.06, \quad b_{\mathrm{GFMS}}=0.07 \pm 0.01$, $m_{\mathrm{GF} 2 \mathrm{MS}}=-0.26 \pm 0.03$, and $b_{\mathrm{GF} 2 \mathrm{MS}}=-0.90 \pm 0.18$.

As with the SFMS, we define the distance above/below these $\mathrm{HI}$ and $\mathrm{H}_{2}$ scaling relations as $\Delta \mathrm{HIMS}$ and $\Delta \mathrm{H}_{2} \mathrm{MS}$, respectively. As with $\triangle \mathrm{SFMS}$, this is a logarithmic ratio in units of dex, as shown below:

$\Delta \mathrm{HIMS}=\log \frac{\mathrm{M}_{\mathrm{HI}}}{\mathrm{M}_{*}}-\log \frac{\mathrm{M}_{\mathrm{HI}, \mathrm{MS}}}{\mathrm{M}_{*}}$

$\Delta \mathrm{H}_{2} \mathrm{MS}=\log \frac{\mathrm{M}_{\mathrm{H}_{2}}}{\mathrm{M}_{*}}-\log \frac{\mathrm{M}_{\mathrm{H}_{2}, \mathrm{MS}}}{\mathrm{M}_{*}}$.

Since galaxies in the SFMS do not correspond to a similarly tight sequence in either the $\mathrm{HI}$ or $\mathrm{H}_{2}$ gas fraction relations, we do not fit Gaussians to determine a width. Instead, we adopt widths of $0.3 \mathrm{dex}(0.2 \mathrm{dex})$ to separate galaxy populations that have $\mathrm{M}_{\mathrm{HI}_{\mathrm{I}}}\left(\mathrm{M}_{\mathrm{H}_{2}}\right)$ values which are similar to the expectation from the simple scaling relation; these widths correspond to the $1 \sigma$ standard deviations of the SFMS population which is detected in each panel. The bottom two panels of Figure 2 show these regions of parameter space. Note that in this work we consistently treat the Hi and molecular gas masses separately. The gas fraction relations of the total (HI + molecular) gas would be qualitatively similar to the HI relations as the gas reservoirs of the SFMS and TZ galaxies are dominated by the $\mathrm{HI}$ component. For a detailed analysis and discussion of scaling relations based on a combined total cold gas mass, see Section 4.2 of Catinella et al. (2018). 

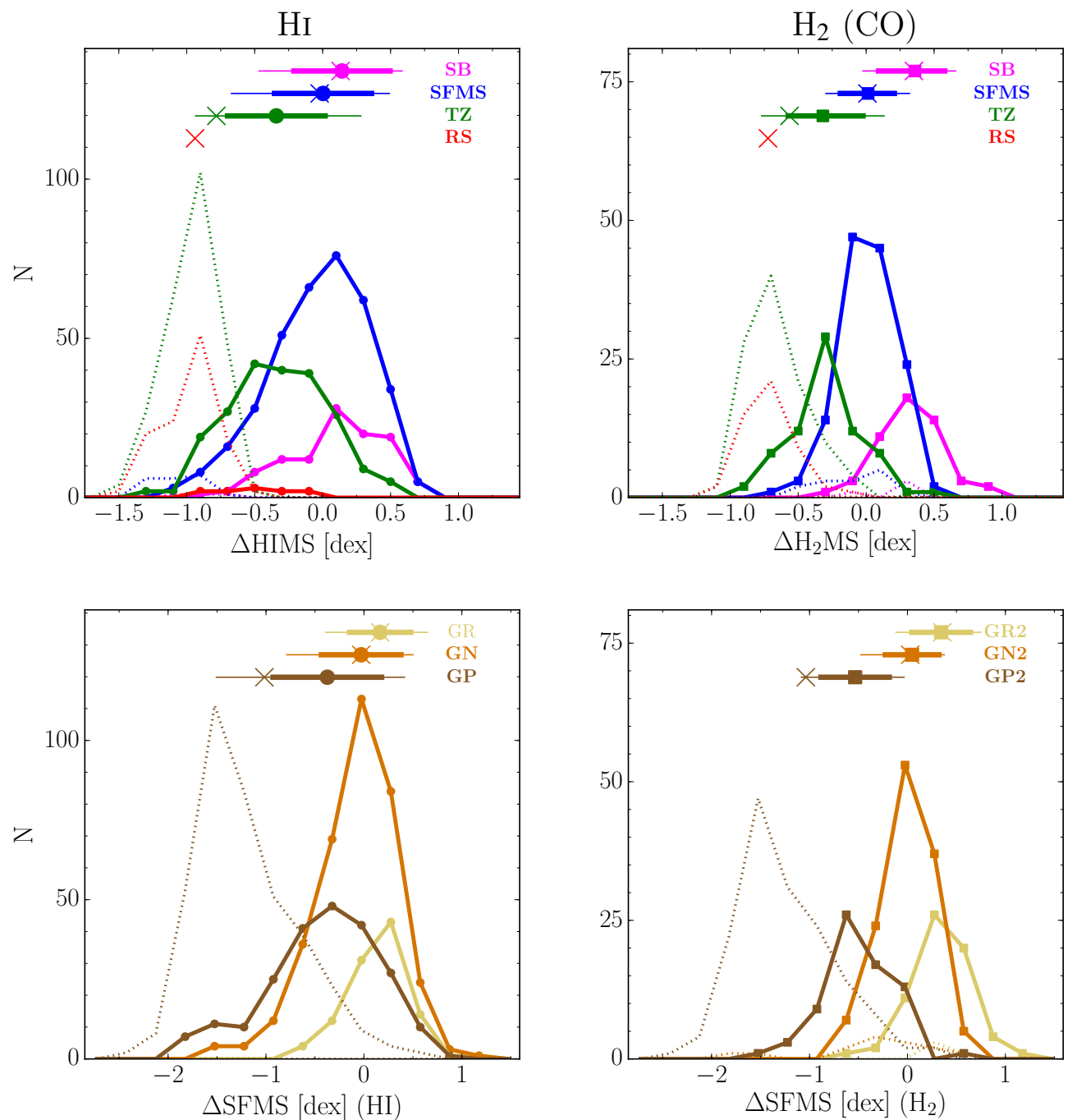

Figure 3. Top panels: histograms of $\Delta \mathrm{HIMS}$ and $\Delta \mathrm{H}_{2} \mathrm{MS}$ for galaxies selected by $\Delta$ SFMS. Bottom panels: histograms of $\Delta \mathrm{SFMS}$ for galaxies selected by $\Delta$ HIMS and $\Delta \mathrm{H}_{2}$ MS. Galaxies which are detected in each population are shown as solid lines, medians as large solid points, thick lines show \pm 1 standard deviations, and thin lines show the 5 th and 95th percentiles of each population (detections only). Non-detections in each population are shown as dotted histograms. Colored crosses indicate the medians of the full samples in each population, including both detections and upper limits of non-detections.
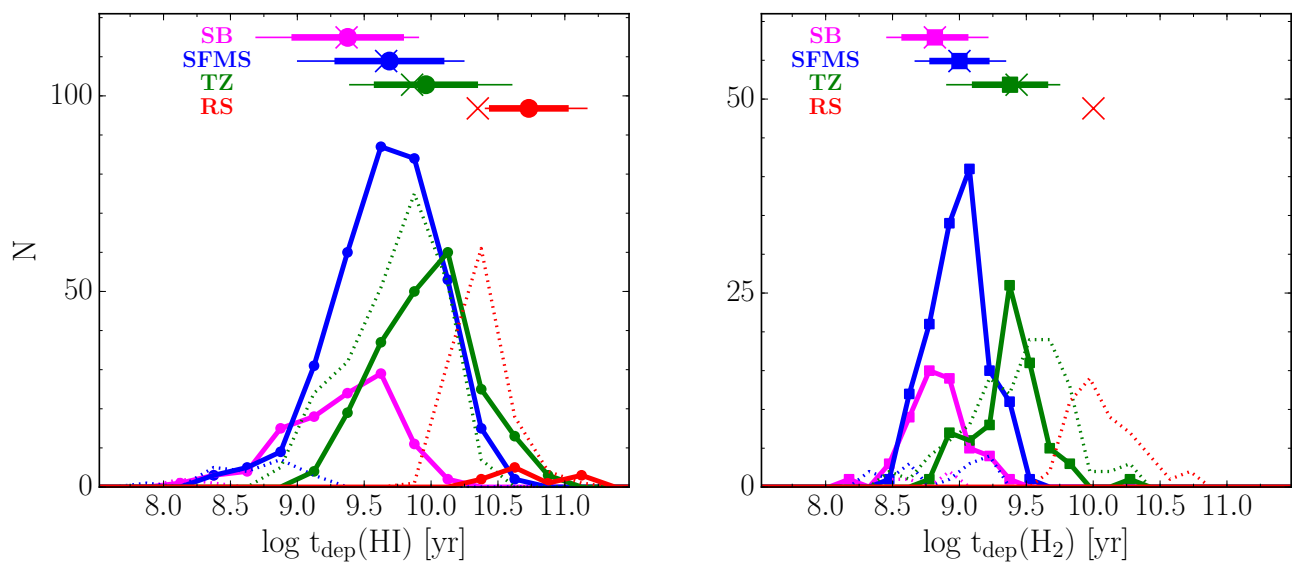

Figure 4. HI (left) and $\mathrm{H}_{2}$ (right) depletion times for SB (magenta), SFMS (blue), and TZ (green) galaxy populations with gas detections (non-detections are shown as dotted lines). Medians of the detections in each population are shown as large symbols with thick $1 \sigma$ error bars and thin lines extending to the 5th and 95th percentiles. Colored crosses show the medians of the full samples in each population, including both detections and upper limits of non-detections. 


\section{COLD GAS ABOVE/BELOW THE SFMS}

Figure 2 demonstrates the complexity of the correspondence between star formation and cold gas content. Galaxies are color coded based on their $\triangle$ SFMS from the $\mathrm{SSFR} \mathrm{M}_{*}$ plot (top two panels). Those within $\pm 0.3 \mathrm{dex}$ (approximately $1 \sigma)$ of the SFMS are shown in blue, and those with higher sSFR are referred to as starbursts (SB) and shown in magenta. The Red Sequence (RS) is typically dominant below sSFR $<-11.8 \mathrm{yr}^{-1}$ (Salim 2014), which corresponds to $\triangle \mathrm{SFMS}<-1.55$ dex for our SFMS, and is shown in red. Between the RS and the SFMS is the Transition Zone (TZ), shown in green. The precise threshold between the TZ and the RS is somewhat arbitrary; we adopt $\triangle \mathrm{SFMS}=-1.55 \mathrm{dex}$ since below that point only $\sim 10 \%(0 \%)$ of the galaxies are detected in $\mathrm{HI}(\mathrm{CO})$. As we are interested in the cold gas properties of TZ galaxies, this RS definition makes our TZ population more meaningful. Our main conclusions would remain unchanged if we adopted variations on this threshold between the TZ and the RS.

On the top left panel of Figure 2, filled circles denote galaxies which have been detected in HI, while colored crosses show sources which do not have HI detections (although still have valid sSFR measurements). The top right panel of Figure 2 shows the same for molecular gas (CO) observations: filled squares show sources with molecular gas detections, while colored crosses denote those which have been observed but not detected (again, these are valid sSFR measurements).

The bottom two panels of Figure 2 show the HI and $\mathrm{H}_{2}$ gas fraction scaling relations, using the $\Delta$ SFMS-selected color scheme. The bottom left panel plots the Hi gas fraction scaling relation, with colored circles showing HI detected galaxies and colored crosses at the upper limits of nondetections. The bottom right panel plots the $\mathrm{H}_{2}$ gas fraction scaling relation, with colored squares showing detections and colored crosses at the upper limits of non-detections. Analogously to the SFMS, here we show the GFMS relation (i.e., fitting the gas fraction scaling relation in $\mathrm{HI}$ and $\mathrm{H}_{2}$ for SFMS galaxies only), and identify galaxies within \pm 0.3 dex (0.2 dex for $\left.\mathrm{H}_{2}\right)$ as gas normal (GN). These widths correspond to $1 \sigma$ standard deviations of the gas fraction distributions of the SFMS population. Galaxies above this sequence are considered gas rich (GR) and below are gas poor (GP).

There is a general correspondence between these two relations in that the majority of galaxies above the SFMS are also above the GFMS (as was also shown in Saintonge et al. 2016), but there is substantial scatter, especially in the HI relations. In particular, a significant number of $\mathrm{TZ}$ galaxies below the SFMS have HI gas fractions which are consistent with (GN) or even above (GR) that of SFMS galaxies. Before discussing specific trends, we first quantify the strength of this correspondence between $\triangle$ SFMS and $\triangle$ HIMS.

\subsection{Correspondence between $\triangle$ SFMS and $\triangle$ HIMS}

First we consider the $\triangle$ SFMS populations (SB, SFMS, TZ, and RS) and plot the distribution of $\triangle$ HIMS for each in the top left panel of Figure 3. We include galaxies with HI detections in the solid histogram (for which statistics are computed) and show the upper limits of non-detections as dotted histograms for reference. These populations all have broad distributions of $\mathrm{HI}_{\mathrm{I}}$ gas fractions with $1 \sigma$ widths of $\sim 0.4$ dex. The average $\triangle \mathrm{HIMS}$ of the SB, SFMS, and TZ populations varies by only $0.15-0.3 \mathrm{dex}$, so there is significant overlap between galaxies above, on, and below the SFMS.

Likewise the top right panel of Figure 3 shows the $\Delta \mathrm{H}_{2} \mathrm{MS}$ distributions for the molecular gas of the same populations. Here the distributions are much tighter $(\sim 0.25 \mathrm{dex})$ but their averages are separated by similar amounts $(0.2-$ 0.3 dex). As was visually apparent in Figure 2, there is a tighter correspondence when comparing SFR with $\mathrm{M}_{\mathrm{H}_{2}}$ than with $\mathrm{M}_{\mathrm{HI}_{\mathrm{I}}}$. As noted in Catinella et al. (2018), galaxies in the SFMS have a narrower range of $\mathrm{H}_{2}$ gas fractions, whereas the HI gas fraction can change by two orders of magnitude. The averages and standard deviations of the $\Delta$ HIMS and $\Delta \mathrm{H}_{2} \mathrm{MS}$ distributions are quantified in Table 1 .

Carrying out a similar selection process in the reverse direction, the bottom left panel of Figure 3 shows the $\triangle$ SFMS distributions of the GFMS-selected populations (GR, GN, GP). Here again the correlation between Hi content and SFR is weak, as all three distributions have significant overlap. The most gas-rich galaxies have the narrowest distribution of $\triangle$ SFMS, suggesting that elevated star formation is very likely given a rich supply of atomic gas. However, even this gas-rich population has a tail that extends to $-1 \sigma_{\mathrm{SF}}$ below the SFMS.

The bottom right panel of Figure 3 shows the $\triangle$ SFMS distributions for the GF2MS-selected populations (GR2, GN2, GP2). These distributions are again tighter than those selected by $\triangle$ HIMS, but not as tight as those in the top right panel. This suggests that the correlation from SFR to $\mathrm{H}_{2}$ content is stronger than the correlation from $\mathrm{H}_{2}$ content to SFR. The averages and standard deviations of these $\triangle$ SFMS distributions for the populations selected by $\mathrm{HI}$ and $\mathrm{H}_{2}$ gas fractions are also given in Table 1.

\subsection{Depletion times and the SFMS}

Galaxies above the SFMS (with higher SFR) are likely to deplete their cold gas reservoirs more quickly than those below the SFMS (with lower SFR). However, given the wide scatter in gas properties below the SFMS, we next compute depletion times $\left(t_{\text {dep }}=M_{\text {gas }} / S F R\right)$ for each galaxy using their $\mathrm{HI}$ and $\mathrm{H}_{2}$ masses. These depletion times represent the timescale for total gas consumption when assuming a very simplistic constant SFR and no gas recycling. Some previous work has suggested that star-forming galaxies are re-fueled on $\sim$ Gyr timescales on average, which complicates this simple picture (Kannappan et al. 2013). Furthermore, the physical extents of the Hi and molecular gas distributions are likely quite different, and our unresolved observations cannot distinguish between gas in the far outskirts (which must first migrate inwards before it can play a role in star formation) and gas in the inner regions (which may be consumed on shorter time scales). Nonetheless, the Hi and $\mathrm{H}_{2}$ depletion times are useful to estimate the evolutionary potential of galaxies on and outside the SFMS. Previous observations of $\mathrm{H}_{2}$ depletion time have shown a near constant value (0.7 Gyr) in SFMS galaxies across cosmic time (Tacconi et al. 2013), while others show a dependence on sSFR such that more star-forming galaxies have shorter depletion times (Saintonge et al. 2011, 2016).

In this work we use the depletion time as a crude esti- 

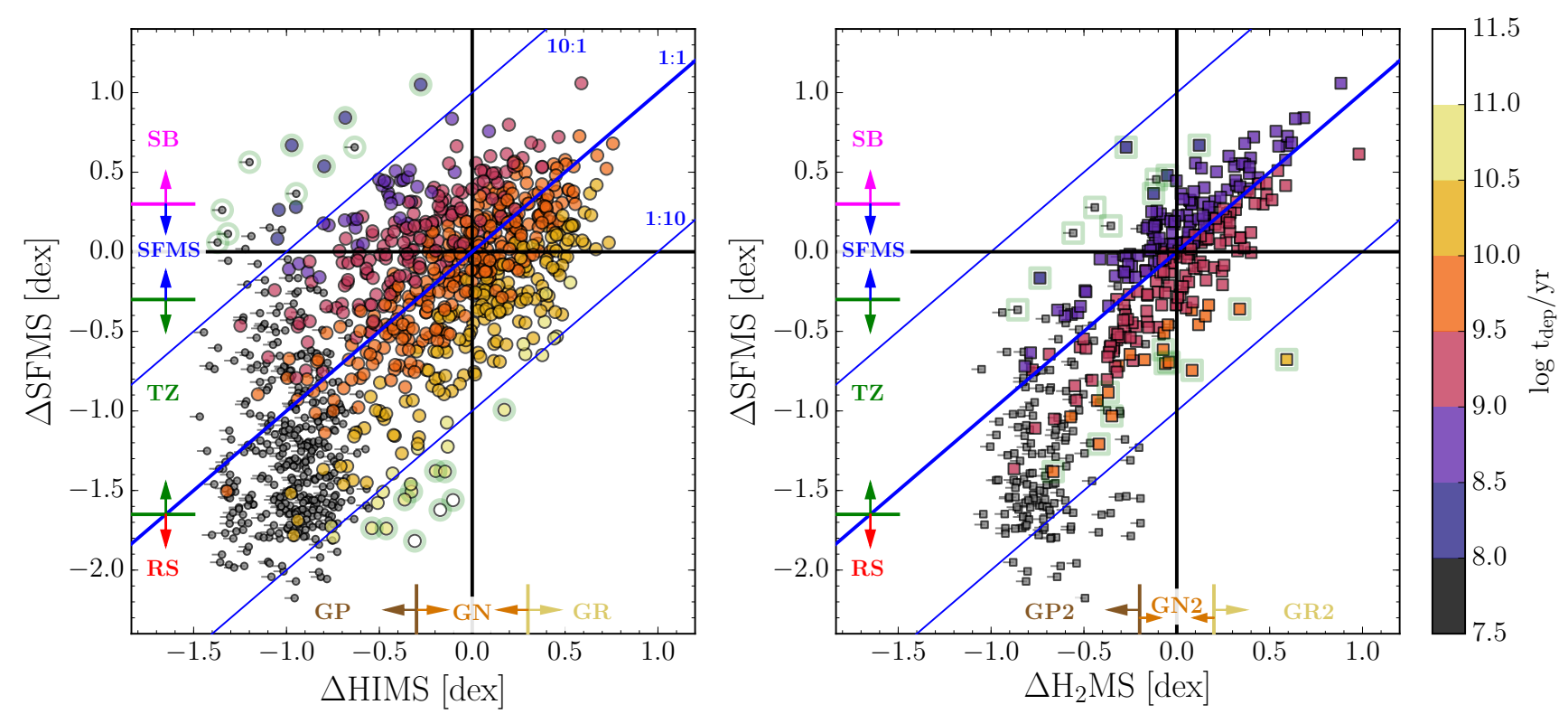

Figure 5. $\triangle$ SFMS is plotted vs. $\triangle \mathrm{HIMS}$ and $\Delta \mathrm{H}_{2} \mathrm{MS}$, color coded by depletion time (using circles for $\mathrm{M}_{\mathrm{H}_{\mathrm{I}}}$ and squares for $\mathrm{M}_{\mathrm{H}_{2}}$, respectively). Regions of $\Delta$ SFMS and $\Delta$ HIMS populations are indicated along each axis. Galaxies with Hi/CO non-detections are shown as small grey symbols at their upper limit $\triangle$ HIMS with short lines extending toward permitted gas fractions. Diagonal lines show 1:1, 10:1, and 1:10 ratios. Note that the depletion time at the origin is different between the two panels $\left(\sim 10^{9.6} \mathrm{yr}\right.$ in $\mathrm{HI}$, and $\sim 10^{9.0} \mathrm{yr}$ in $\mathrm{H}_{2}$ ). The ten longest and ten shortest depletion time galaxies in each panel are surrounded by light green halos and are discussed further in Section 3.3 (see also Figure 6).

Shortest Hi depletion times:
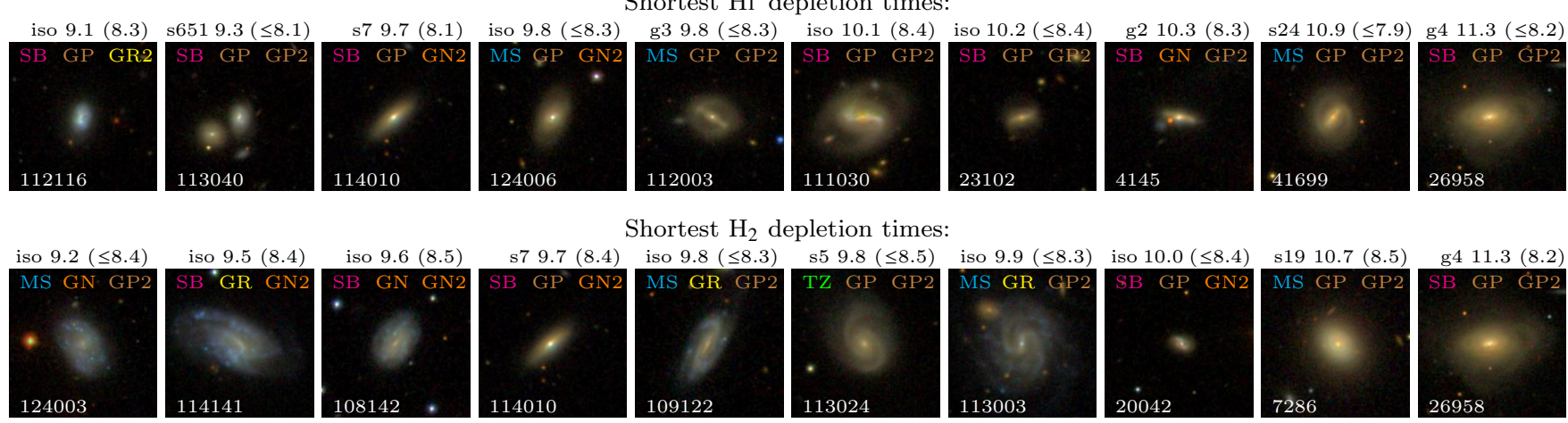

Shortest $\mathrm{H}_{2}$ depletion times:
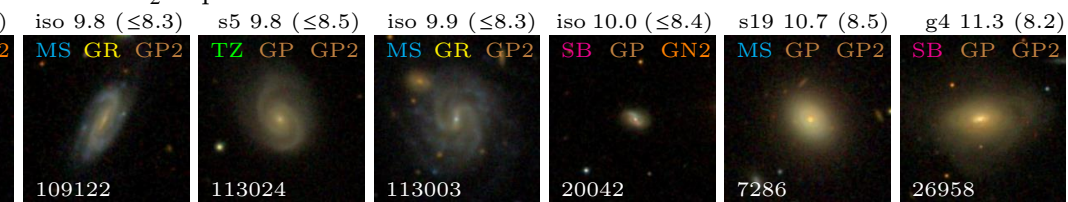

Longest Hi depletion times:
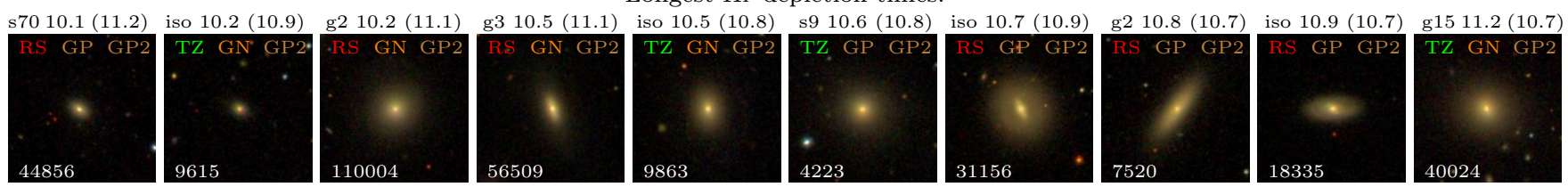

Longest $\mathrm{H}_{2}$ depletion times:
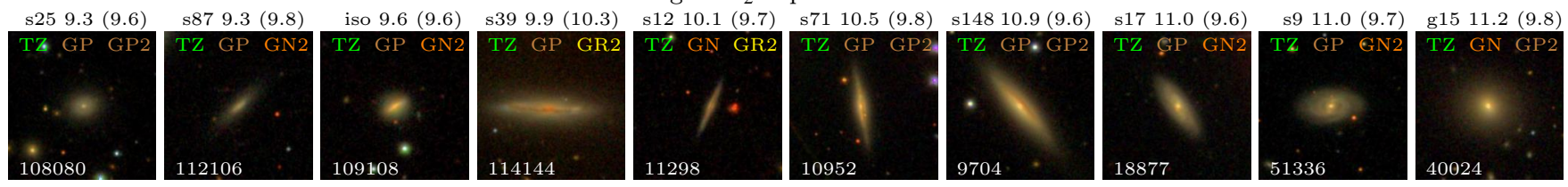

Figure 6. SDSS thumbnail images $\left(80^{\prime \prime}\right.$ on a side) are shown of galaxies with the extreme $\mathrm{HI}_{\mathrm{I}}$ and $\mathrm{H}_{2}$ depletion times, ordered by increasing stellar mass from left to right. Above each thumbnail is its environmental identity (isolated central or satellite/group central with the number of group members), the logarithm of its stellar mass (in solar units) and the logarithm of its depletion time (in years and in parentheses). Overlaid on each image are its $\triangle \mathrm{SFMS}, \Delta \mathrm{HIMS}$, and $\Delta \mathrm{H}_{2} \mathrm{MS}$ categories, and its xGASS ID. 
Table 1. Population sizes (detections and non-detections), averages and standard deviations (of detections only), and medians (of detections and non-detections) are given for each selection. $<\Delta \mathrm{HIMS}>$ and $<\Delta \mathrm{H}_{2} \mathrm{MS}>$ are computed for SFMS-selected populations (SB, SFMS, TZ, RS), and $<\Delta$ SFMS $>$ is computed for $\Delta$ HIMS- and $\Delta \mathrm{H}_{2}$ MS-selected populations (GR, GN, GP). When fewer than half of the galaxies in a selected population are detected, medians are not computed.

Hi $\quad \mathrm{H}_{2}$

\begin{tabular}{|c|c|c|c|c|c|c|c|c|}
\hline$\triangle \mathrm{SFMS}$ & $\mathrm{N}_{\mathrm{d}}$ & $\left(\mathrm{N}_{\mathrm{nd}}\right)$ & $<\Delta \mathrm{HIMS}>$ & $<\Delta \mathrm{HIMS}>_{\mathrm{med}}$ & $\mathrm{N}_{\mathrm{d}}$ & $\left(\mathrm{N}_{\mathrm{nd}}\right)$ & $<\Delta \mathrm{H}_{2} \mathrm{MS}>$ & $<\Delta \mathrm{H}_{2} \mathrm{MS}>_{\text {med }}$ \\
\hline SB & 107 & (3) & $+0.10(0.35)$ & +0.13 & 52 & (4) & $+0.33(0.24)$ & -0.20 \\
\hline SFMS & 349 & (20) & $-0.03(0.36)$ & -0.02 & 136 & (14) & $+0.01(0.20)$ & -0.02 \\
\hline $\mathrm{TZ}$ & 211 & $(246)$ & $-0.33(0.36)$ & - & 73 & (105) & $-0.30(0.27)$ & - \\
\hline $\mathrm{RS}$ & 11 & $(117)$ & $-0.53(0.28)$ & - & 0 & $(50)$ & - & - \\
\hline$\Delta \mathrm{HIMS}$ & $\mathrm{N}_{\mathrm{d}}$ & $\left(\mathrm{N}_{\mathrm{nd}}\right)$ & $<\Delta \mathrm{SFMS}>$ & $<\Delta \mathrm{SFMS}>_{\text {med }}$ & $\mathrm{N}_{\mathrm{d}}$ & $\left(\mathrm{N}_{\mathrm{nd}}\right)$ & $<\Delta \mathrm{SFMS}>$ & $<\Delta \mathrm{SFMS}>_{\text {med }}$ \\
\hline $\operatorname{GR}(2)$ & 106 & (0) & $+0.15(0.31)$ & +0.17 & 65 & (4) & $+0.33(0.30)$ & +0.36 \\
\hline $\mathrm{GN}(2)$ & 350 & (0) & $-0.08(0.41)$ & -0.03 & 126 & (14) & $-0.00(0.27)$ & +0.03 \\
\hline $\operatorname{GP}(2)$ & 222 & (386) & $-0.43(0.55)$ & -1.02 & 70 & (155) & $-0.51(0.35)$ & - \\
\hline
\end{tabular}

mate of the evolutionary pace of a galaxy: those with shorter depletion times are likely to be more rapidly evolving and transforming (i.e., consuming a significant fraction of their gas supply on short timescales), while those with longer depletion times are evolving more slowly. The left panel of Figure 4 shows histograms of Hi depletion times for each $\triangle$ SFMS population (SB, SFMS, TZ, RS) using the same color-coding as previous figures. Within each population, average Hi depletion times based on Hi detections are shown with large symbols with $1-\sigma$ error bars as well as 5 th and 95 th percentiles. Crosses show medians within each population, using both detections and non-detections.

Galaxies in the xGASS SFMS (in blue on Figure 4) span a wide range of $\mathrm{HI}$ depletion times varying from 0.8-40 Gyr. The average Hi depletion time is $\sim 6.2$ Gyr with a scatter of 0.6 dex. In the TZ below the SFMS (plotted in green), galaxies span a similar dynamic range of $\mathrm{HI}$ depletion times (from 7-70 Gyr, one order of magnitude), with an average of $~ 9.7$ Gyr. Above the SFMS, SB galaxies (plotted in magenta) again have a larger dynamic range (1.3-130 Gyr) but a shorter average Hi depletion time of $\sim 2.9 \mathrm{Gyr}$.

The right panel of Figure 4 shows histograms of $\mathrm{H}_{2}$ depletion times for the same $\triangle$ SFMS populations with the same color-coding. Within the SFMS, the dynamic range of $\mathrm{H}_{2}$ depletion times is similar that in Hi, but offset to shorter times, as shown in the right panel of Figure 4, spanning only $0.3-3$ Gyr, with an average of $\sim 1.1$ Gyr and a scatter of 0.49 dex. As with $\mathrm{HI}$, SB galaxies have shorter $\mathrm{H}_{2}$ depletion times (average of $0.8 \mathrm{Gyr}$ ), and galaxies in the $\mathrm{TZ}$ have longer $\mathrm{H}_{2}$ depletion times (average of $2.8 \mathrm{Gyr}$ ).

Combining all of the cold gas and SFR information, we plot $\triangle$ SFMS vs. $\Delta$ HIMS and $\Delta \mathrm{H}_{2} \mathrm{MS}$ in Figure 5 , in a similar way to Figure 11 of Schiminovich et al. (2010). These plots show the general trend for galaxies above (below) the average $\mathrm{HI}$ and $\mathrm{H}_{2}$ scaling relations to have positive (negative) $\triangle \mathrm{SFMS}$, and they also demonstrate the significant amount of scatter in this trend. Points in Figure 5 are color coded by their gas depletion time, and SFMS- and GFMS-selected populations are labeled along the axes.

\subsection{Galaxies with extreme depletion times}

Given the broad correspondence between $\Delta$ HIMS and $\triangle \mathrm{SFMS}$ in Figure 5, we take a closer look at those which deviate from this general trend. That is, we examine galaxies with the most extreme depletion times to explore what factors may contribute to their departure from the main relation. It is important to note that a galaxy's SFR is expected to stochastically fluctuate, so a galaxy which currently exhibits an extreme depletion time may be experiencing a brief fluctuation in its SFR (increase or decrease) and not a permanent transition. These fluctuations can have particularly strong effects on the lowest mass galaxies in our sample.

First, we select the 10 galaxies with the shortest HI depletion times and indicate their locations with light green halos in the top left corner of the left panel of Figure 5. We include $\mathrm{HI}$ non-detections in this selection, as these translate directly to upper limits on depletion times, and note that these short Hi depletion time galaxies are all in the SFMS or SB populations. SDSS thumbnail images are shown for these galaxies in the top row of Figure 6. We also include the environmental identity for each galaxy from the Yang et al. (2007) DR7 group catalog (discussed further in Section 4), and do not include the 16 galaxies from our sample which lack environmental identities largely due to their proximity to survey edges.

These short Hi depletion time galaxies have (potentially completely) diminished gas reservoirs but unexpectedly strong star formation. As seen in their SDSS thumbnails, at least half show strong bars or ring features, suggesting they may have more efficient star formation than an otherwise similar galaxy without a bar. These short depletion time galaxies are likely to be the most rapidly evolving systems. Without an additional supply of gas, their SFR will soon decrease and they may drop back down to (or even below) the 1:1 line on Figure 5.

Similarly, we select the 10 galaxies with the shortest $\mathrm{H}_{2}$ depletion times and indicate their positions in the right panel of Figure 5. These are found largely in the SB and SFMS, but one is in the TZ. Thumbnails from SDSS of this population are shown in the second row of Figure 6, and they have similar morphologies to the galaxies with the longest Hi depletion times. Only two galaxies (xGASS 114010 and 26958) are common to both categories: xGASS 114010 has a strong bulge and is a satellite in a group of $\mathrm{N}=7$; xGASS 26985 is a central in a group of $\mathrm{N}=4$ and shows a dramatic system of shells/rings suggesting a recent interaction.

At the other extreme, we consider the 10 galaxies with 

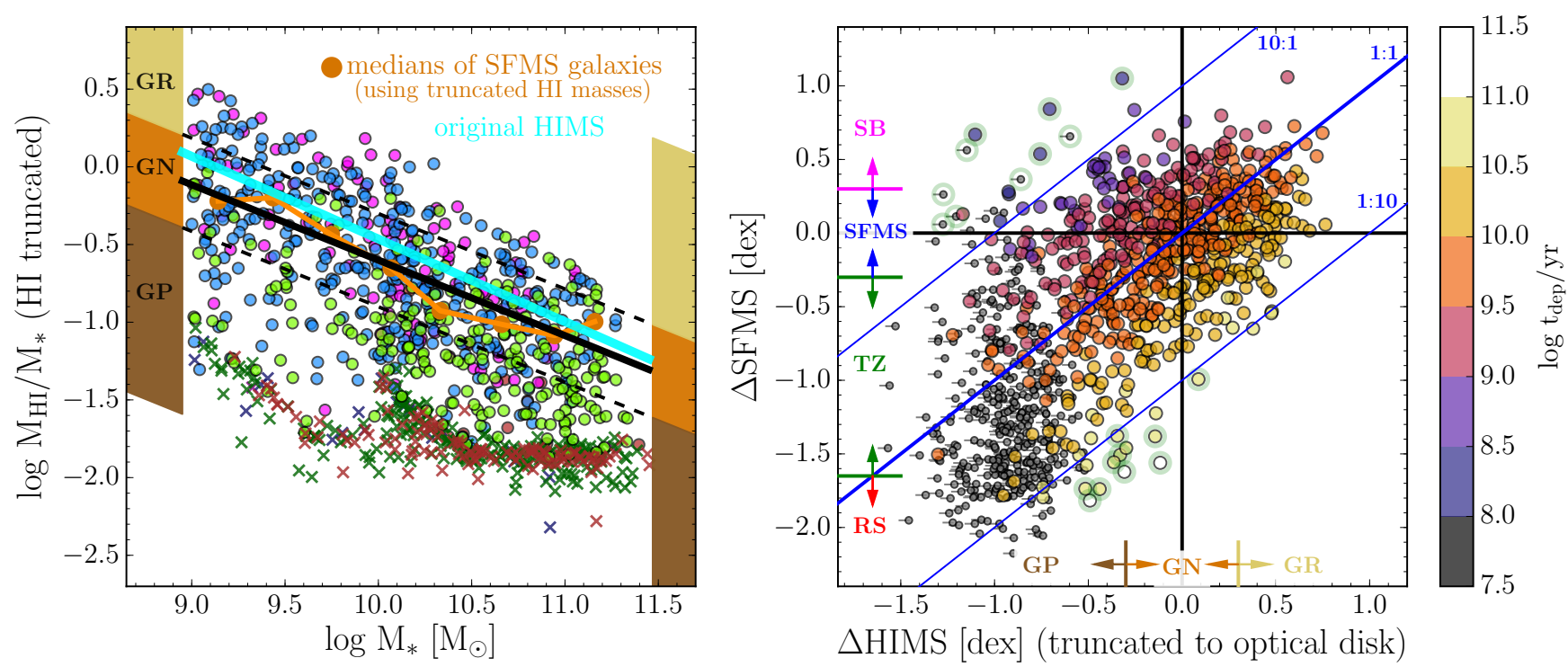

Figure 7. Left panel: Hi gas-fraction scaling relation is plotted using Hi masses truncated within the the optical disk, as described in Section 3.4. Orange points and black line indicate the median relation for SFMS galaxies, compared to the original relation from Figure 2 shown in cyan. Right panel: truncated Hi masses are used to reproduce the left panel of Figure 5, using the updated HIMS from the left panel. While subtle, there is a small systematic offset towards lower values of $\Delta$ HIMS, but the same appearance overall.

the longest Hi depletion times, which are also indicated with light green halos in the bottom right corner of the left panel of Figure 5, and their thumbnails are shown in the third row of Figure 6. Here we require Hi detections to compute meaningful depletion times, as upper limits on Hi masses also yield upper limits on HI depletion times. Without a detection, the true depletion time could be significantly smaller and much less extreme. This population of long Hi depletion time galaxies (with good HI detections) is found in the TZ and RS.

These galaxies have a significantly larger Hi reservoir than expected from their SFR, so the atomic gas may be inaccessible, lingering below a density threshold, or experiencing too much shear or dispersion to trigger star formation. This inaccessibility could mean that the gas has arrived with high angular momentum from a minor merger, so has not yet collapsed enough to form a proportionate amount of star formation. Our Hi observations are unresolved, so the neutral gas has not been localized within the galaxy, and may be significantly more extended than the stellar components (perhaps analogously to the sample identified and observed by Geréb et al. 2016, 2018), but interferometric observations of their Hi would be required to confirm this scenario. These galaxies are all fairly massive $\left(\mathrm{M}_{*} \geq 10^{10} \mathrm{M}_{\odot}\right)$ and have strong bulges, but at least four show (hints of) disks as well. Galaxies with substantial Hi reservoirs like these are candidates for possible rejuvenation (i.e., a return to the SFMS) if they are able to access their full gas supply for star formation. At their current star formation rates, these long-depletiontime galaxies are some of the most slowly evolving galaxies. They are unlikely to move significantly within Figure 5 and represent a moderately static gas-rich population.

Analogously, the longest $\mathrm{H}_{2}$ depletion time population is selected in the same way and displayed in the 4 th row of Figure 6. Note in particular that GASS 40024 has both an extreme $\mathrm{HI}$ depletion time and an extreme $\mathrm{H}_{2}$ depletion time, and is a satellite in a group of $\mathrm{N}=15$ galaxies. This long $\mathrm{H}_{2}$ depletion time population also includes galaxies at lower masses and are all found in the TZ. Here the number of edge-on galaxies with visible absorption from dust lanes suggests that some members of this population may have under-estimated SFRs. Our SFR is based on both the direct UV emission from young stars and the re-processed IR emission from dust absorption, but the edge-on systems with strongly visible dust lanes may be more extincted than our SFR calibration assumes. If the SFR estimates of edge-on galaxies are under-estimated, then their depletion times will be artificially increased.

\subsection{Predicting gas location from scaling relations and predicting $\mathrm{HI}$ masses within optical disk}

One might consider the role that the spatial location of cold gas within the galaxies in our sample plays in determining their star formation histories and depletion times. For example, gas-rich TZ galaxies may remain gas-rich (with long depletion times) if their remaining gas is largely located in their outskirts and only slowly migrates to the inner regions to participate in active star formation. Accounting for potential differences in $\mathrm{HI}$ and stellar distributions would require resolved $\mathrm{HI}$ observations, but even without those we can employ simple scaling relations to predict the amount of Hi mass contained within the optical disk. In this way we can estimate the depletion time considering only the gas which is predicted to be contained within the optical disk.

We use the Hi size-mass relation from Wang et al. (2016) to predict an Hi size using only the Hi mass of each galaxy in our sample. We also adopt a simple exponential profile for the radial Hi mass distribution. Using the $90 \%$ Petrosian radius from the SDSS $r$ image, we integrate the exponential profile within this truncation radius to determine the Hi mass contained within the optical disk. This is a simple approach and relies on the assumption that these 
galaxies all follow the Hi size-mass relation and all have the same profile shape. Still, these simple truncated Hi masses will give an indication of the potential impact that the spatial distribution of Hi could have on our results.

The left panel of Figure 7 shows an updated version of the Hi gas fraction scaling relation, originally presented in Figure 2, but now using the Hi masses truncated within the optical disk. As expected, this overall reduction in Hi masses results in a slightly lower median relation than with the full Hi masses, with a very weak change in slope. The right panel of Figure 7 plots $\triangle$ SFMS vs $\Delta$ HIMS for these truncated HI masses. When compared with the left panel of Figure 5, no significant differences are observed. Since the truncated HI masses are always smaller than the total Hi masses, there is again a systematic shift toward lower values of $\Delta \mathrm{HIMS}$. This does not indicate that the spatial distribution of the gas is unimportant, but rather suggests that there are no significant effects revealed by this truncation of an assumed HI profile within the optical disk. Resolved observations of the HI gas would be required to advance this question further.

\section{UN-QUENCHED GALAXY POPULATIONS BELOW THE SFMS}

Using the populations described in Figure 5, we next focus on the galaxies found in the TZ between the SFMS and the RS. Here we refer to "quenched" galaxies as those with SFR measurements low enough to be consistent with no active star formation and such small cold gas reservoirs as to be undetectable. We will consider the types of evolutionary scenarios and pathways that TZ galaxies may follow. First, however, it is important to note that galaxies in the $\mathrm{TZ}$ with cold gas detections have on average $\sim 0.35$ dex longer $\mathrm{HI}$ and $\mathrm{H}_{2}$ depletion times than those in the SFMS. This is visible in Figure 4, which also makes clear the significant overlap between both populations. Of the $\sim 400$ galaxies in the TZ, $\sim 50 \%$ are detected in $\mathrm{Hi}$ and $40 \%$ of these $\mathrm{HI}$ detections are comparably or more gas-rich than typical SFMS galaxies (i.e., are GN or GR). This suggests that a significant fraction of the $\mathrm{TZ}$ galaxies are not actively quenching or rapidly evolving towards the RS. The remaining TZ galaxies without $\mathrm{HI}$ detections may be a more rapidly evolving/quenching population, but the TZ galaxies with Hi detections can sustain their current SFR longer than galaxies currently in the SFMS!

While all galaxies in the TZ could naively be considered "quenched" based on their sSFR alone, we are interested in separating the TZ into meaningful populations that may evolve on different pathways. We begin by separating TZ galaxies by their environmental identity as satellite or central galaxies, since we expect these populations to evolve through different mechanisms. We use the SDSS DR7 group catalog of Yang et al. (2007) to divide our sample into satellite members of groups, central members of groups, and isolated central galaxies, and we first consider the satellite galaxy population.

\subsection{Satellite galaxies in the $\mathrm{TZ}$}

There are 160 satellite galaxies from xGASS in the TZ, of which 67 have Hi detections. The left panel of Figure 8 shows the $\triangle$ HIMS vs. $\triangle$ SFMS plot for all satellites, with medians plotted in bins of $\triangle$ SFMS. Compared with central galaxies (in groups and in isolation), satellites have median $\Delta$ HIMS values which are smaller both on and below the SFMS (above the SFMS, the central and satellite populations are indistinguishable). The median offset in $\Delta$ HIMS between the HI-detected TZ satellite and central galaxies is $\sim 0.47 \mathrm{dex}$, which is larger than the analogous offset in the SFMS ( $\sim .10 \mathrm{dex})$. Quantitatively, the distributions of $\triangle$ HIMS for HI-detected central and satellite galaxies in the TZ are different at the $98.8 \%$ level $(p=0.012)$ using a twotailed $p$-value KS test. This reduced gas content of satellite galaxies is broadly consistent with environmental stripping/quenching scenarios, where a reduction of gas content precedes a decrease in star formation activity.

While the $\triangle$ HIMS distributions of central and satellite galaxies in the TZ show statistically significant differences, it is difficult to physically interpret any differences in $\Delta$ HIMS alone since it implicitly includes a dependence on $\mathrm{M}_{*}$ (present in the Hi gas fraction shown in Figure 2). In order to quantify this apparent quenching of satellite galaxies in a more physical sense, we instead consider the distributions of their HI depletion times (using only galaxies with Hi detections). In the TZ, a KS test of the distributions of Hi depletion times for central and satellite galaxies produces a two-sided $\mathrm{p}$-value of $p=0.13$, implying these samples are only weakly distinct from each other.

To dig deeper into the significant trends identified in $\Delta$ HIMS, Figure 9 shows the HI depletion times for TZ galaxies now separated into three bins of stellar mass. In all bins, the distributions of Hi depletion times for satellite and central TZ galaxies have similar widths of $\sim 0.4$ dex. In the lowest mass bin (with the highest Hi detection fraction), satellite TZ galaxies have median Hi depletion times of $10^{9.6} \mathrm{yr}$, while central $\mathrm{TZ}$ galaxies have a median of $10^{9.8} \mathrm{yr}$. This difference is not very large and a KS test on these two distributions of depletion times yields only $p=0.26$. The same galaxies have $\triangle$ HIMS distributions which are different by $p=0.07$, which is somewhat more significant. The middle and high mass bins show even smaller differences between TZ satellites and centrals, so this trend appears to be driven by the galaxies at lower mass.

The reason why Hi depletion times show less of a difference than $\triangle$ HIMS is most likely because changes in SFR and $\mathrm{HI}$ content cancel each other and/or increase the scatter. As shown in Figure 8, central and satellite galaxies are different at nearly fixed $\triangle$ SFMS, but their difference relative to the diagonal dashed line (i.e., constant depletion time) is inevitably less significant.

While this difference in Hi depletion times is small $(p=0.012)$, a systematically lower HI content in satellites at fixed star formation rate (i.e., shorter depletion times) than centrals is in line with a simple evolutionary picture where satellite quenching is driven primarily by gas stripping. In this scenario, satellite galaxies in the TZ (especially those at low mass) have had their gas reservoirs reduced more rapidly and their star formation is still declining to match. This points towards active stripping of gas being more important in the evolution of satellites than central galaxies, though the large scatter may indicate multiple evolutionary paths also for the satellite population. 

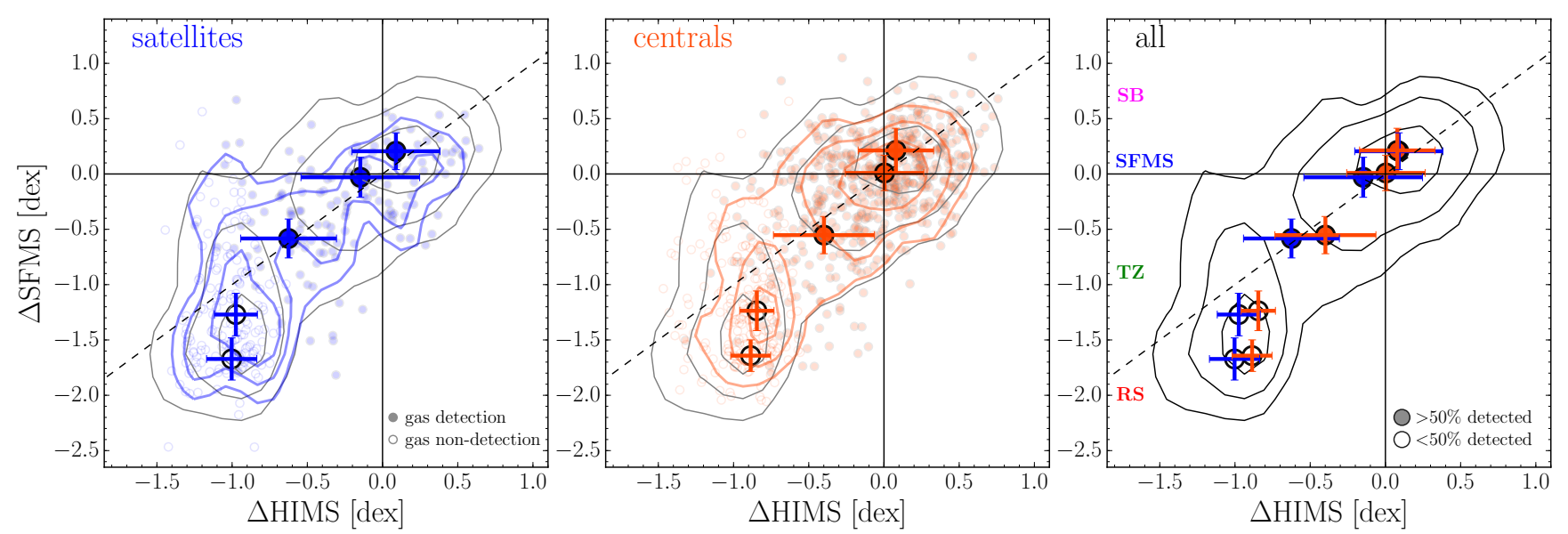

Figure 8. Each panel shows $\triangle$ HIMS vs. $\triangle$ SFMS, for satellite galaxies (left panel, blue), central galaxies (middle panel, red), and the total population (right panel). Contours and medians in bins of $\Delta$ SFMS are shown for each population, with 25 th/75th percentile error bars; the unity line is shown with dashes. Large median points are filled if more than half are detected in Hi. Satellite galaxies in the $\mathrm{TZ}$ are offset to lower median $\Delta$ HIMS than central galaxies; a KS test on these two distributions produces $p=0.012$ implying they are significantly different from each other.

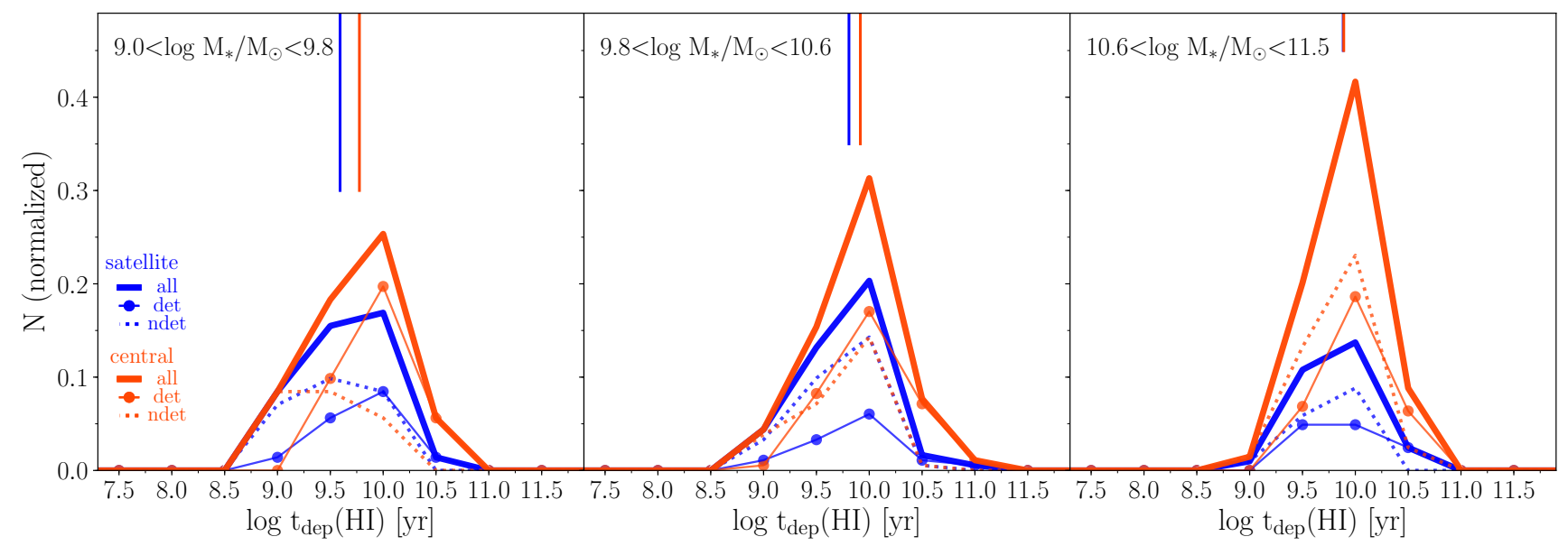

Figure 9. Normalized histograms of Hi depletion time for TZ galaxies which are centrals (red) and satellites (blue), divided into three bins of stellar mass. Thick lines show histograms of all of the (HI) detected and non-detected galaxies, thin lines show only detections, and dotted lines show only non-detections. Vertical lines are drawn at the median depletion time for each population (including detections and non-detections). In the lowest mass bin the satellites $(\mathrm{N}=12)$ have shorter depletion times than centrals $(\mathrm{N}=25)$ and a $\mathrm{KS}$ test between these two distributions gives $p=0.26$ which is a weak difference. The middle and high mass bins show less significant differences in their Hi depletion times.

\subsection{Central galaxies in the TZ}

There are 297 central galaxies from xGASS in the TZ, of which 158 have Hi detections. Galaxies in the TZ span a similarly wide range of Hi depletion times compared with those in the SFMS or above it. As discussed in Section 4.1, the smaller gas reservoirs of satellite galaxies in the TZ is consistent with a picture of environmental effects driving them into the TZ. In this section we now focus on the central galaxies, which we expect to be less affected by their environment. Here we combine all central galaxies (those in groups/clusters and those in isolation) into a single sample for better statistics. This yields a sample of 297 central galaxies in the TZ. We also carry out the following analysis on subsets of isolated only and group central galaxies only, and find no significant differences from the results discussed below.

To explore this population, we divide the central galaxies in the $\mathrm{TZ}$ into three roughly equal-sized populations based on their (HI) depletion times. "Typical" centrals have $\mathrm{t}_{\text {dep }}$ between $\sim 5$ and $\sim 11$ Gyr, and represent the middle trintile of the distribution. Those with longer depletion times are considered "slow," and shorter are considered "fast." We also note that this population of central $\mathrm{TZ}$ galaxies with "typical" Hi depletion times is found along the 1:1 line shown in Figure 5, the "fast" are found above the unity line, and the "slow" below.

We test for possible trends with a few fundamental observed properties of the TZ centrals in these "fast," "typical," and "slow" populations. With regard to their environment, we consider the density of nearby neighbors and their halo 


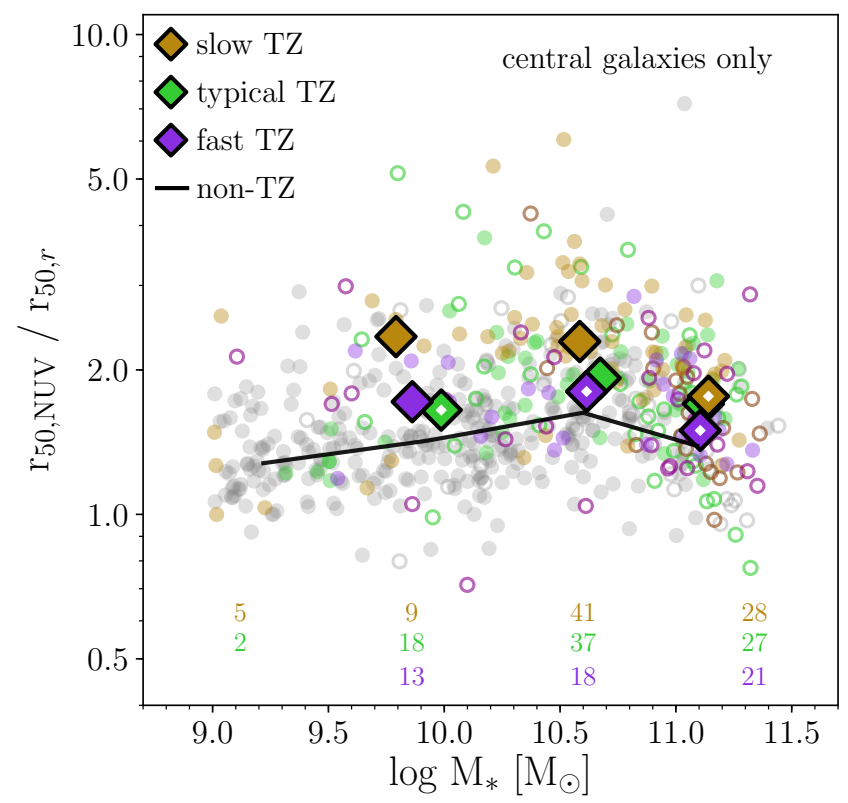

Figure 10. Only central galaxies are plotted, using different colors for those in the TZ with short HI depletion times (fast, purple), average depletion times (typical, green), and long (slow, brown). Grey symbols indicate central galaxies outside of the TZ. Solid symbols denote Hi detections, and open show upper limits of non-detections. Large diamonds show medians (including detections and non-detections) in bins of stellar mass (when fewer than $50 \%$ are detected, the diamonds are open). Numbers at the bottom indicate how many galaxies are in each binned median. The black line connects medians of the non-TZ central galaxies. TZ central galaxies show a trend with the UV-to-optical size ratio such that those with longer (slower) depletion times have larger ratios.

mass as given by abundance matching in the group catalog of Yang et al. (2007). When using the density of galaxies per projected $\mathrm{Mpc}^{2}$ within the 7 th nearest neighbor, we find no significant difference between these three populations of $\mathrm{TZ}$ centrals. Similarly, no trends are found with the halo masses. Both of these results suggest that the gas content in TZ centrals is not strongly affected by their environment, and that this population may evolve through a variety of channels.

Looking internally, we also look for trends with average stellar surface density, $\mu_{*}$, defined as $\mathrm{M}_{*} /\left(2 \pi \mathrm{R}_{50, z}^{2}\right)$, where the $z$ filter half-light radius $R_{50, z}$ is measured in kpc. All three populations of $\mathrm{TZ}$ centrals have larger average values than non-TZ central galaxies, which is consistent with the typical morphologies seen below the SFMS (e.g., Salim et al. 2007). However, we find no significant trends as a function of $\mu_{*}$. This further shows the diversity and scatter within this population.

The only meaningful trend we identify appears when considering the ratio of UV and optical size of the TZ centrals. We use the ratio of the half-light radius measured in NUV to the half-light radius in the optical $r$ filter, including only $\sim 75 \%$ of galaxies that have been resolved in the GALEX images (i.e., $r>5^{\prime \prime}$ ). Here the three TZ central populations show a consistent trend: those with the longest Hi depletion times also have the highest average UV-to-optical size ra-
Slow TZ (larger UV-to-optical size ratio):

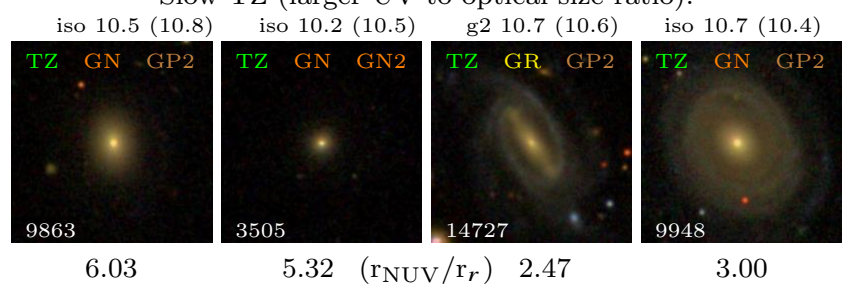

Fast TZ (smaller UV-to-optical size ratio):

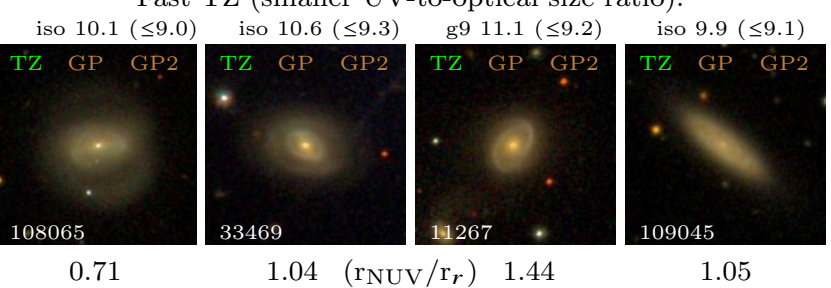

Figure 11. SDSS color images of example central galaxies from the TZ, selected for their slow (top row) or fast (bottom row) Hi depletion times. TZ central galaxies with long depletion times typically show star formation activity in their outskirts and have a larger UV-to-optical size ratio, while those with short depletion times more often have central star formation and small ratios of UV-to-optical size. Galaxies are labeled as in Figure 6, with their environmental identity, stellar mass, and Hi depletion time. The UV-to-optical size ratio $\left(\mathrm{r}_{\mathrm{NUV}} / \mathrm{r}_{r}\right)$ of each galaxy is listed underneath its thumbnail.

tios, and the smallest Hi depletion times have the smallest UV-to-optical size ratios.

Figure 10 shows this UV-to-optical size ratio vs. stellar mass for central galaxies in our sample. Those in the TZ are plotted in colors corresponding to their depletion time; all non-TZ central galaxies are shown as grey dots. There is a weak trend evident with stellar mass such that more massive galaxies have larger UV-to-optical size ratio. Additionally, most of the central galaxies with the largest UV-to-optical size ratios are found in the TZ. However, most intriguing is the trend amongst the $\mathrm{TZ}$ galaxies such that those with longer depletion time (i.e., "slow") have larger typical UV-tooptical size ratios. This is most clearly visible in the stellar mass bin centered around $10^{10.5} \mathrm{M}_{\odot}$, where there are more galaxies of each population included in the median. For a visual example, Figure 11 shows sample images of TZ galaxies in the "slow" (with large UV-to-optical size ratio) and "fast" (with small UV-to-optical size ratio) subsets. In particular, GASS 3505 was identified by Geréb et al. (2016) as a prototypical example of an "HI-excess" galaxy - a population which has large Hi reservoirs but insignificant amounts of star formation (Geréb et al. 2018).

This correlation between depletion time and UV-tooptical size ratio suggests that the star formation in the "slow" TZ galaxies is more radially extended than in typical galaxies. It may mean that the excess Hi reservoir is also more spatially extended and inaccessible to star formation. An inaccessible Hi reservoir may lead to low star formation efficiency; $\sim 90 \%$ of the "slow" TZ central galaxies have depletion times longer than a Hubble time (and $\sim 15 \%$ are more than twice as long as a Hubble time). Intriguingly, for the $\sim 40 \%$ of $\mathrm{TZ}$ galaxies with molecular observations there is no correlation between the molecular and atomic gas depletion times, and all three populations have average molec- 
ular gas depletion times of $\sim 3$ Gyr. The apparent tightness of the relation between $\triangle$ SFMS and $\Delta \mathrm{H}_{2} \mathrm{MS}$ (shown in the right panel of Figure 5) supports the fact that SFR proceeds whenever molecular gas is available (see, e.g., Schruba et al. 2011); the inaccessible Hi may be in an extended low-density configuration which prevents it from converting to molecular gas and forming stars. These "slow" galaxies may still be forming stars (albeit slowly) long after the galaxies in the SFMS have exhausted their gas, fully quenched, and joined the RS.

\section{DISCUSSION}

Galaxies in the TZ below the SFMS do not appear to make up a unified population, as many external and internal mechanisms can drive galaxies into (or out of) this so-called "green valley." Compared to SFMS galaxies, TZ galaxies have a similar range of $\mathrm{HI}$ gas fractions and longer depletion times, and are found across all environments, providing a large range of available evolutionary pathways.

When considering the role of external environmental effects, we have demonstrated that satellite galaxies with low $\triangle$ HIMS (see Section 4.1) may be examples of some TZ galaxies which have experienced a cessation of star formation following the ram pressure stripping of their gas by a cluster environment. Vulcani et al. (2015) also find a weak connection between environment and quenched galaxies, but also identify a population of galaxies with intermediate optical colors which are not rapidly quenched but instead are experiencing declining SFH over long time scales (>1 Gyr).

Our sample of $\mathrm{TZ}$ galaxies have average $\mathrm{HI}$ depletion times of 10 Gyr (see Section 3.2), suggesting that this population is not rapidly moving toward the RS through secular evolution alone. This population of long depletion time TZ galaxies is in contrast with the expectations from other studies of the stellar populations of TZ galaxies. For example, while Smethurst et al. (2015) find evidence for multiple evolutionary paths through the $\mathrm{TZ}$, their scenario only requires 1-1.5 Gyr for a galaxy to transit the TZ. Similarly, using the EAGLE hydrodynamic simulation, Trayford et al. (2016) selected TZ galaxies based on their $u-r$ colors and found that they spend less than 2 Gyr transiting between the SFMS and RS. This apparently rapid quenching and disagreement with our findings is largely a result of selecting $\mathrm{TZ}$ galaxies (and determining their SFRs) based only on optical colors. As effectively demonstrated by Figure 4 in Salim (2014), TZ and $\mathrm{RS}$ galaxies have indistinguishable optical colors and it is difficult to make meaningful distinctions between these populations without UV observations.

Another formation scenario for TZ galaxies is that they have depleted their gas reservoirs and are fading onto the RS. The results of Schawinski et al. (2014) support the idea of multiple evolutionary pathways through the TZ, but argue that the star formation rates are responding to changes in their (un-observed) gas supplies. They claim that latetype TZ galaxies have had their cosmic gas supply cut off, and that early-type TZ galaxies have recently experienced a rapid quenching as a result of the destruction of their gas supply by a possible major merger. (We found no dependence of any of our relationships on $\mu_{*}$, a proxy for morphology, as discussed in Section 4.2). Bremer et al. (2018) also argue that an insufficient gas supply is responsible for galaxies rapidly ( 1-2 Gyr) evolving through the TZ. While our work does confirm that $\mathrm{TZ}$ galaxies have lower average HI gas fractions than those in the SFMS, the range of gas fractions is actually larger in the TZ, and their long gas depletion times ( 10 Gyr on average) are not consistent with rapid quenching scenarios.

When selecting TZ galaxies based on UV and optical observations, others have also found $\mathrm{TZ}$ galaxies still forming stars at a sustainable rate. In particular, Fang et al. (2012) identified a population of "extended star-forming early-type galaxies" (ESF-ETGs) which were selected using their global photometry on a UV-optical color-magnitude diagram. While consistent with TZ in a global sense, these ESF-ETGs look red and morphologically early-type at their centers but their outskirts are blue and show UV emission from young stars. Fang et al. (2012) suggest that this population of TZ galaxies may persist for several Gyrs before fading into the RS, consistent with our results.

Studies of the gas content in star-forming galaxies at higher redshifts have identified similar trends to those discussed in this work. Notably, the Plateau de Bure high-z Blue Sequence Survey (PHIBSS, Genzel et al. 2015; Tacconi et al. 2018) observed molecular gas masses in 32 galaxies at $z=1.2$ and 2.2 with stellar masses above $10^{10.4} \mathrm{M}_{\odot}$, and found that their molecular gas fraction generally decreases with time and that their molecular gas depletion times increase further below the SFMS, independent of stellar mass. More recently, the low redshift $(\mathrm{z}=0.5-0.8)$ sample of PHIBSS2 galaxies between $10^{10}<\mathrm{M}_{*} / \mathrm{M}_{\odot}<10^{11.4}$ showed that this trend for molecular gas depletion time to increase below the SFMS also continues towards lower redshifts (Freundlich et al. 2019). These relations are also consistent with the results of Saintonge et al. (2012, 2017), who used the COLD GASS and xCOLD GASS samples (which are also used in this work and consist of direct $\mathrm{CO}$ observations rather than indirect gas indicators used at higher redshifts) to determine that the molecular gas depletion time increases by $\sim 0.3$ dex between galaxies in the SFMS and the TZ. Our findings that local galaxies below the SFMS have longer HI depletion times fits naturally into this picture, and we have explored a few of the features and mechanisms at play in this evolution.

Many evolutionary paths lead through the TZ, and today's TZ galaxies appear to be able to evolve in almost any direction in Figure 5. As a population, our TZ galaxies do not appear to represent an intermediate stage between the SFMS and the RS, but rather are a diverse population, as seen most clearly through observations of their cold gas content.

\section{SUMMARY}

In this work we have used the xGASS and xCOLD GASS samples to explore the cold gas properties of galaxies in the $\mathrm{TZ}$ below the SFMS. Our main findings are:

- Galaxies in the SFMS have large scatter in Hi gas fraction scaling relation, but tighter in $\mathrm{H}_{2}$. There is no corresponding "HI main sequence" of galaxies in the SFMS.

- Compared with galaxies in the SFMS, the HI depletion times of galaxies in the $\mathrm{TZ}$ are longer, suggesting it is 
not a uniformly rapidly quenching/evolving population, and evolves more slowly than the SFMS.

- Satellite galaxies in the TZ show a trend to be more gas poor than $\mathrm{TZ}$ centrals, especially in terms of $\Delta$ HIMS. This trend is weaker when measured in $\mathrm{HI}$ depletion time and appears to originate from the lowest mass galaxies $\left(\mathrm{M}_{*} / \mathrm{M}_{\odot}<10^{9.8}\right)$, which is generally consistent with environmental stripping processes removing the gas.

- Central galaxies in the TZ show a trend between their HI depletion time and UV-to-optical size ratios, suggesting that they have remained gas rich as a result of inefficient star formation proceeding slowly in their outer disks. Visual inspection of this population shows a variety of disk galaxies with strong bulges and blue outer disks.

In order to further improve our understanding of the evolutionary pathways available to galaxies in the TZ, we need enhanced spatial and temporal resolution of their star formation activity. Given the spatial distributions of UV emission discussed in Section 4.2, followup imaging in $\mathrm{H} \alpha$ or UV would allow us to better understand if star formation in TZ galaxies is proceeding in different ways than in the SFMS. Re-constructing the star formation histories of TZ galaxies would be invaluable in determining whether they have been a long-lived TZ population or have recently experienced a decline in SFR.

Better separating the mass contributions from the disk (star-forming, associated with gas reservoir) and the bulge (passive) in the xGASS galaxies would also provide a clearer picture of the evolutionary context of the TZ (e.g., Cook et al. 2019). Bulge growth can contribute to a galaxy's decline from the SFMS to the TZ, and in cases where the depletion times of the bulge and disk can be mapped separately (e.g., Lin et al. 2017), it is clear that bulge quenching and disk quenching are important and somewhat independent processes which drive galaxies away from the SFMS. A better separation of bulge and disk masses will help clarify these evolutionary pathways.

\section{ACKNOWLEDGEMENTS}

We thank Toby Brown, Katinka Geréb, and Robin Cook for helpful discussions, and the anonymous referee for their careful reading and thoughtful suggestions which have helped improve this work.

SJ, BC, and LC acknowledge support from the Australian Research Council's Discovery Project funding scheme (DP150101734). Parts of this research were conducted by the Australian Research Council Centre of Excellence for All Sky Astrophysics in 3 Dimensions (ASTRO 3D), through project number CE170100013. LC is the recipient of an Australian Research Council Future Fellowship (FT180100066) funded by the Australian Government.

This research has made use of NASA's Astrophysics Data System, and also the NASA/IPAC Extragalactic Database (NED), which is operated by the Jet Propulsion Laboratory, California Institute of Technology, under contract with the National Aeronautics and Space Administration. This research has also made extensive use of the amaz- ingly invaluable Tool for OPerations on Catalogues And Tables (TOPCAT ${ }^{2}$, Taylor 2005).

Funding for SDSS-III has been provided by the Alfred P. Sloan Foundation, the Participating Institutions, the National Science Foundation, and the U.S. Department of Energy Office of Science. The SDSS-III web site is http://www.sdss3.org/.

SDSS-III is managed by the Astrophysical Research Consortium for the Participating Institutions of the SDSSIII Collaboration including the University of Arizona, the Brazilian Participation Group, Brookhaven National Laboratory, Carnegie Mellon University, University of Florida, the French Participation Group, the German Participation Group, Harvard University, the Instituto de Astrofisica de Canarias, the Michigan State/Notre Dame/JINA Participation Group, Johns Hopkins University, Lawrence Berkeley National Laboratory, Max Planck Institute for Astrophysics, Max Planck Institute for Extraterrestrial Physics, New Mexico State University, New York University, Ohio State University, Pennsylvania State University, University of Portsmouth, Princeton University, the Spanish Participation Group, University of Tokyo, University of Utah, Vanderbilt University, University of Virginia, University of Washington, and Yale University.

\section{REFERENCES}

Abazajian, K. N., Adelman-McCarthy, J. K., Agüeros, M. A., et al. 2009, ApJS, 182, 543-558

Bouché, N., Dekel, A., Genzel, R., et al. 2010, ApJ, 718, 1001

Bremer, M. N., Phillipps, S., Kelvin, L. S., et al. 2018, MNRAS, 476,12

Brinchmann, J., Charlot, S., White, S. D. M., et al. 2004, MNRAS, 351, 1151

Catinella, B., Schiminovich, D., Kauffmann, G., et al. 2010, MNRAS, 403, 683

Catinella, B., Schiminovich, D., Cortese, L., et al. 2013, MNRAS, 436,34

Catinella, B., Saintonge, A., Janowiecki, S., et al. 2018, MNRAS, Chabrier, G. 2003, PASP, 115, 763

Cook, R. H. W., Cortese, L., Catinella, B., et al. 2019, MNRAS, 490, 4060

Cortese, L., \& Hughes, T. M. 2009, MNRAS, 400, 1225

Cortese, L. 2012, A\&A, 543, A132

Daddi, E., Dickinson, M., Morrison, G., et al. 2007, ApJ, 670, 156

Elbaz, D., Daddi, E., Le Borgne, D., et al. 2007, A\&A, 468, 33

Fang, J. J., Faber, S. M., Salim, S., Graves, G. J., \& Rich, R. M. 2012, ApJ, 761, 23

Freundlich, J., Combes, F., Tacconi, L. J., et al 2019, A\&A, 622, 105

Genzel, R., Tacconi, L. J., Lutz, D., et al. 2015, ApJ, 800, 20

Geréb, K., Catinella, B., Cortese, L., et al. 2016, MNRAS, 462, 382

Geréb, K., Janowiecki, S., Catinella, B., Cortese, L., \& Kilborn, V. 2018, MNRAS, 476, 896

Janowiecki, S., Catinella, B., Cortese, L., et al. 2017, MNRAS, 466, 4795

Kannappan, S., Stark, D., Eckert, K., et al. 2013, ApJ, 777, 42

Lilly, S. J., Carollo, C. M., Pipino, A., Renzini, A., \& Peng, Y. 2013, ApJ, 772, 119

Lin, L., Belfiore, F., Pan, H.-A., et al. 2017, ApJ, 851, 18

Magdis, G. E., Elbaz, D., Daddi, E., et al. 2010, ApJ, 714, 1740

2 http://www.starlink.ac.uk/topcat/ 
Martin, D. C., Fanson, J., Schiminovich, D., et al. 2005, ApJ, 619, L1

Martin, D. C., Wyder, T. K., Schiminovich, D., et al. 2007, ApJS, 173,342

Morrissey, P., Conrow, T., Barlow, T. A., et al. 2007, ApJS, 173, 682

Noeske, K. G., Faber, S. M., Weiner, B. J., et al. 2007, ApJ, 660, L47

Saintonge, A., Kauffmann, G., Kramer, C., et al. 2011, MNRAS, 415,32

Saintonge, A., Tacconi, L. J., Fabello, S., et al. 2012, ApJ, 758, 73

Saintonge, A., Catinella, B., Cortese, L., et al. 2016, MNRAS, 462,1749

Saintonge, A., Catinella, B., Tacconi, L. J., et al. 2017, ApJS, 233,22

Salim, S., Rich, R. M., Charlot, S., et al. 2007, ApJS, 173, 267

Salim, S. 2014, Serbian Astronomical Journal, 189, 1

Salim, S., Lee, J. C., Janowiecki, S., et al. 2016, ApJS, 227, 2

Schawinski, K., Urry, C. M., Simmons, B. D., et al. 2014, MNRAS, 440,889

Schiminovich, D., Catinella, B., Kauffmann, G., et al. 2010, MNRAS, 408, 919

Schruba, A., Leroy, A. K., Walter, F., et al. 2011, AJ, 142, 37

Smethurst, R. J., Lintott, C. J., Simmons, B. D., et al. 2015, MNRAS, 450, 435

Tacconi, L. J., Neri, R., Genzel, R., et al. 2013, ApJ, 768, 74

Tacconi, L. J., Genzel, R., Saintonge, A., et al. 2018, ApJ, 853, 179

Taylor, M. B. 2005, Astronomical Data Analysis Software and Systems XIV, 347, 29

Trayford, J. W., Theuns, T., Bower, R. G., et al. 2016, MNRAS, 460, 3925

Vulcani, B., Poggianti, B. M., Fritz, J., et al. 2015, ApJ, 798, 52

Wang, J., Kauffmann, G., Overzier, R., et al. 2011, MNRAS, 412, 1081

Wang, J., Koribalski, B. S., Serra, P., et al. 2016, MNRAS, 460, 2143

Whitaker, K. E., van Dokkum, P. G., Brammer, G., \& Franx, M. 2012, ApJ, 754, L29

Whitaker, K. E., Franx, M., Leja, J., et al. 2014, ApJ, 795, 104

Woo, J., Dekel, A., Faber, S. M., et al. 2013, MNRAS, 428, 3306

Wright, E. L., Eisenhardt, P. R. M., Mainzer, A. K., et al. 2010, AJ, 140, 1868-1881

Wyder, T. K., Martin, D. C., Schiminovich, D., et al. 2007, ApJS, 173,293

Yang, X., Mo, H. J., van den Bosch, F. C., et al. 2007, ApJ, 671, 153

This paper has been typeset from a $\mathrm{T}_{\mathrm{E}} \mathrm{X} / \mathrm{L}_{\mathrm{E}} \mathrm{X}$ file prepared by the author. 